\title{
Newsvendor characterizations for one-warehouse multi- retailer inventory systems with descrete demand under the balance assumption
}

\section{Citation for published version (APA):}

Dogru, M. K., Kok, de, A. G., \& Houtum, van, G. J. J. A. N. (2013). Newsvendor characterizations for onewarehouse multi-retailer inventory systems with descrete demand under the balance assumption. Central European Journal for Operations Research and Economics, 21(3), 541-559. https://doi.org/10.1007/s10100-0120246-7

DOI:

10.1007/s10100-012-0246-7

Document status and date:

Published: 01/01/2013

\section{Document Version:}

Accepted manuscript including changes made at the peer-review stage

\section{Please check the document version of this publication:}

- A submitted manuscript is the version of the article upon submission and before peer-review. There can be important differences between the submitted version and the official published version of record. People interested in the research are advised to contact the author for the final version of the publication, or visit the DOI to the publisher's website.

- The final author version and the galley proof are versions of the publication after peer review.

- The final published version features the final layout of the paper including the volume, issue and page numbers.

Link to publication

\footnotetext{
General rights

- You may freely distribute the URL identifying the publication in the public portal. follow below link for the End User Agreement:

www.tue.nl/taverne

\section{Take down policy}

If you believe that this document breaches copyright please contact us at:

openaccess@tue.nl

providing details and we will investigate your claim.
}

Copyright and moral rights for the publications made accessible in the public portal are retained by the authors and/or other copyright owners and it is a condition of accessing publications that users recognise and abide by the legal requirements associated with these rights.

- Users may download and print one copy of any publication from the public portal for the purpose of private study or research.

- You may not further distribute the material or use it for any profit-making activity or commercial gain

If the publication is distributed under the terms of Article $25 \mathrm{fa}$ of the Dutch Copyright Act, indicated by the "Taverne" license above, please 


\title{
Newsvendor characterizations for one-warehouse multi-retailer inventory systems with discrete demand under the balance assumption
}

\author{
Mustafa K. Doğru • A. G. de Kok • \\ G. J. van Houtum
}

(C) Springer-Verlag 2012

\begin{abstract}
This paper considers a one-warehouse multi-retailer inventory system that faces discrete stochastic demand of the customers. Under the so-called balance assumption (also known as the allocation assumption), base stock policies are optimal. Our main contribution is to show that the optimal base stock levels satisfy newsvendor characterizations, which are in terms of inequalities, and to extend the newsvendor equalities known for the continuous demand model. These characterizations are appealing because they (i) are easy to explain to nonmathematical oriented people like managers and MBA students, (ii) contribute to the understanding of optimal control, (iii) help intuition development by providing direct relation between cost and optimal policy parameters.
\end{abstract}

Keywords Multi-echelon · One-warehouse multi-retailer system · Stochastic discrete demand $\cdot$ Newsvendor characterizations

\footnotetext{
M. K. Doğru (ه)

Alcatel-Lucent Bell Labs, 600 Mountain Avenue, Murray Hill, NJ 07974, USA

e-mail: mustafa.dogru@alcatel-lucent.com
}

A. G. de Kok · G. J. van Houtum

Industrial Engineering and Innovation Sciences, Technische Universiteit Eindhoven,

P.O. Box 513, 5600 MB Eindhoven, The Netherlands
A. G. de Kok
e-mail: a.g.d.kok@tue.nl
G. J. van Houtum
e-mail: g.j.v.houtum@tue.nl 


\section{Introduction}

This paper considers a one-warehouse multi-retailer inventory system (also known as a distribution system) under periodic review and centralized control. There are $N$ retailers supplied by shipments from a warehouse (indexed as stock point 0 ), which in return orders from an exogenous supplier with ample stock. Retailers face stochastic and independent demands of the customers. Demands in different periods are i.i.d., discrete nonnegative random variables. Excess demand is backlogged and penalty costs are incurred. Leadtimes of orders (between the supplier and the warehouse), and shipments (between the warehouse and the retailers) are fixed. Costs consist of linear holding and penalty costs; there are no fixed costs. The objective is to minimize the average inventory holding and penalty costs of the system in the long-run.

A cornerstone paper on multi-echelon inventory systems is by Clark and Scarf (1960). The authors developed a dynamic programming formulation for the inventory control of an $N$-stage serial system (serial structures are a special case of distribution systems where each stage has a single predecessor and successor stage). They showed that the resulting $N$ dimensional dynamic program (DP) can be analyzed by solving $N$ single-dimensional DPs recursively (we refer to this result as the decomposition property). Further, they characterized the optimal policy where each stage follows an echelon base stock policy. Clark and Scarf (1960) were the first to consider the inventory control problem in a distribution system facing continuous demands. However, it was not possible to decompose these systems due to the so-called allocation problem: How to allocate the physical stock at the warehouse among all stock points in the system in a given period?

The optimal policy for the inventory control of a one-warehouse multi-retailer inventory system is unknown, and expected to be complex; Doğru (2006) provides numerical analysis of the optimal policy for a few instances that supports the claim. However, an assumption, known as the balance assumption, leads to the decomposition property and the full characterization of the optimal policy. This key assumption is the relaxation of the physical constraint that the inventory positions of the retailers just after the shipment decisions are greater than or equal to the inventory positions prior to these decisions. Other interpretations of the balance assumption are

- allowing negative quantities to be shipped to the retailers,

- permitting immediate return (with no leadtime) of stock at any retailer to the warehouse at no cost,

- allowing the lateral transhipment (shipments between the retailers) of stock with the leadtime of the receiving retailer at no cost. (In our setting, lateral transhipment has a broader meaning. It does not only imply the shipment of on-hand stock from one retailer to the other, but also includes shipment of stock from one retailer's pipeline inventory to the other.)

The balance assumption is essential to obtain structural results and it has been used extensively in the literature, see Axsäter (2003) and the references therein. Moreover, to the best of our knowledge, all heuristics developed for one-warehouse multi-retailer inventory systems under periodic review are based on the balance assumption. 
The one-warehouse multi-retailer inventory system under the balance assumption (referred to as the relaxed model) is well studied in the literature; see Eppen and Schrage (1981); Federgruen and Zipkin (1984a,b); Chen and Zheng (1994) and Diks and de Kok (1998). For the relaxed model, the optimal ordering and allocation policies have been shown to be echelon base stock policy and myopic allocation, respectively.

In this paper, we specifically analyze the one-warehouse multi-retailer inventory system with discrete demands. The one-warehouse multi-retailer model has clear-cut applications in inventory, manufacturing (Rogers and Tsubakitani 1991) and hierarchial production planning context (Wijngaard 1982 and de Kok 1989). It is also utilized to study delayed production differentiation (Lee and Tang 1997 and Aviv and Federgruen 2001). Moreover, the model is related to general kanban systems, but without a capacity restriction at the warehouse, see de Kok and Fransoo (2003, pp. 653-654). Discrete demand processes are important because they make it possible to handle positive probability mass at any point in the demand distribution, particularly at zero. This is highly important in case of intermittent demand. Further, it is relevant to discrete part manufacture-to-order systems, and inventory systems where a unit is equivalent to a bulk and indivisible quantity like a container or a pallet. A possible application area in the former systems is spare parts inventory control where mean demands are generally low.

Our main contribution is to show that the optimal base stock levels in a onewarehouse multi-retailer inventory system under the balance assumption (the relaxed model) satisfy newsvendor inequalities. Newsvendor characterizations show a direct relation between the probability of no-stockout at a retailer as a consequence of an optimal base stock level and the cost parameters (holding and penalty costs) in the from of equalities or inequalities. Newsvendor characterizations are appealing because they

- are easy to explain to nonmathematical oriented people like managers and MBA students,

- contribute to the understanding of optimal control,

- help intuition development by providing direct relation between cost and optimal policy parameters.

For example, our newsvendor inequalities show that the warehouse becomes a crossdocking point if a retailer has zero added value and an infinite support for the demand distribution. This insight is not obtained directly by the formulas of Chen and Zheng (1994) nor of Federgruen and Zipkin (1984a). Moreover, we specify the properties of the allocation functions that lead to the newsvendor characterizations.

Newsvendor characterizations for multi-echelon inventory systems, see van Houtum (2006) for a review, were first derived by van Houtum and Zijm (1991) who showed that the optimal base stock levels in a serial system satisfy newsvendor equations. Similar characterizations were identified for serial systems with fixed replenishment intervals by van Houtum et al. (2007). For serial systems where materials flow from one stock point to another in fixed batches (which is a generalization of Clark and Scarf model where fixed batch quantities equal to one at each stock point), echelon $(R, Q)$ policies have been proven to be optimal by Chen (2000). Doğru et al. (2008) identified that the optimal reorder levels satisfy newsvendor characterizations. Diks and de Kok (1998) showed that the optimal base stock levels in a one-warehouse 
multi-retailer inventory system with continuous demands satisfy newsvendor equations. Our newsvendor inequalities extend the newsvendor characterizations derived for continuous demands to discrete demands.

Finally, the results are used to develop an efficient computational scheme for the determination of optimal base stock levels in the relaxed model.

The rest of the paper is organized as follows. In Sect. 2, we introduce the model and review main results known for one-warehouse multi-retailer inventory systems. Our results are given in Sect. 3. We first present our results for nondecreasing optimal allocation functions. Newsvendor inequalities and the algorithm for the computation of an optimal policy are discussed in Sects. 3.1 and 3.2, respectively. We conclude briefly in Sect. 4. The proofs omitted in the main body can be found in the Appendix.

\section{Preliminaries and notation}

Time is divided into periods of equal length and the following sequence of events takes place during a period: (i) inventory levels are observed and the current period's ordering/shipment decisions are made considering the arrival of the orders/shipments given before (at the beginning of the period), (ii) orders/shipments arrive following their respective leadtimes (at the beginning of the period), (iii) demand occurs, (iv) holding and penalty costs are assessed on the period ending inventory and backorder levels (at the end of the period).

We go over some basic definitions for the sake of completeness. Echelon stock of a stock point is the stock on hand at that point plus in transit to or on hand at any successor stock point minus the backorders of external customers. Echelon inventory position of a stock point is the echelon stock of that stock point plus all the stock that is in-transit to that stock point. The basic notation for this study is given in Table 1 .

In the remainder of this section, the ordering and allocation decisions and their impact on the costs will be discussed. Further, the optimal ordering and allocation policies under the balance assumption will be reviewed. This lays the ground for our results in Sect. 3. (cf. Section 8.6 in Zipkin 2000).

\subsection{Dynamics of the system}

The total cost of the system at the end of an arbitrary period $t$ is

$$
h_{0}\left(\hat{I}_{0}(t)-\sum_{i \in J} \hat{I}_{i}(t)\right)+\sum_{i \in J}\left(h_{0}+h_{i}\right) \hat{I}_{i}^{+}(t)+\sum_{i \in J} p_{i} \hat{I}_{i}^{-}(t),
$$

where $a^{+}=\max \{0, a\}$ and $a^{-}=-\min \{0, a\}$ for $a \in \mathbb{R}$. Substituting $\hat{I}_{i}(t)=\hat{I}_{i}^{+}(t)-$ $\hat{I}_{i}^{-}(t)$ first, rearranging the terms, and then using the identity $\hat{I}_{i}^{+}(t)=\hat{I}_{i}(t)+\hat{I}_{i}^{-}(t)$ yields 
Table 1 Notation

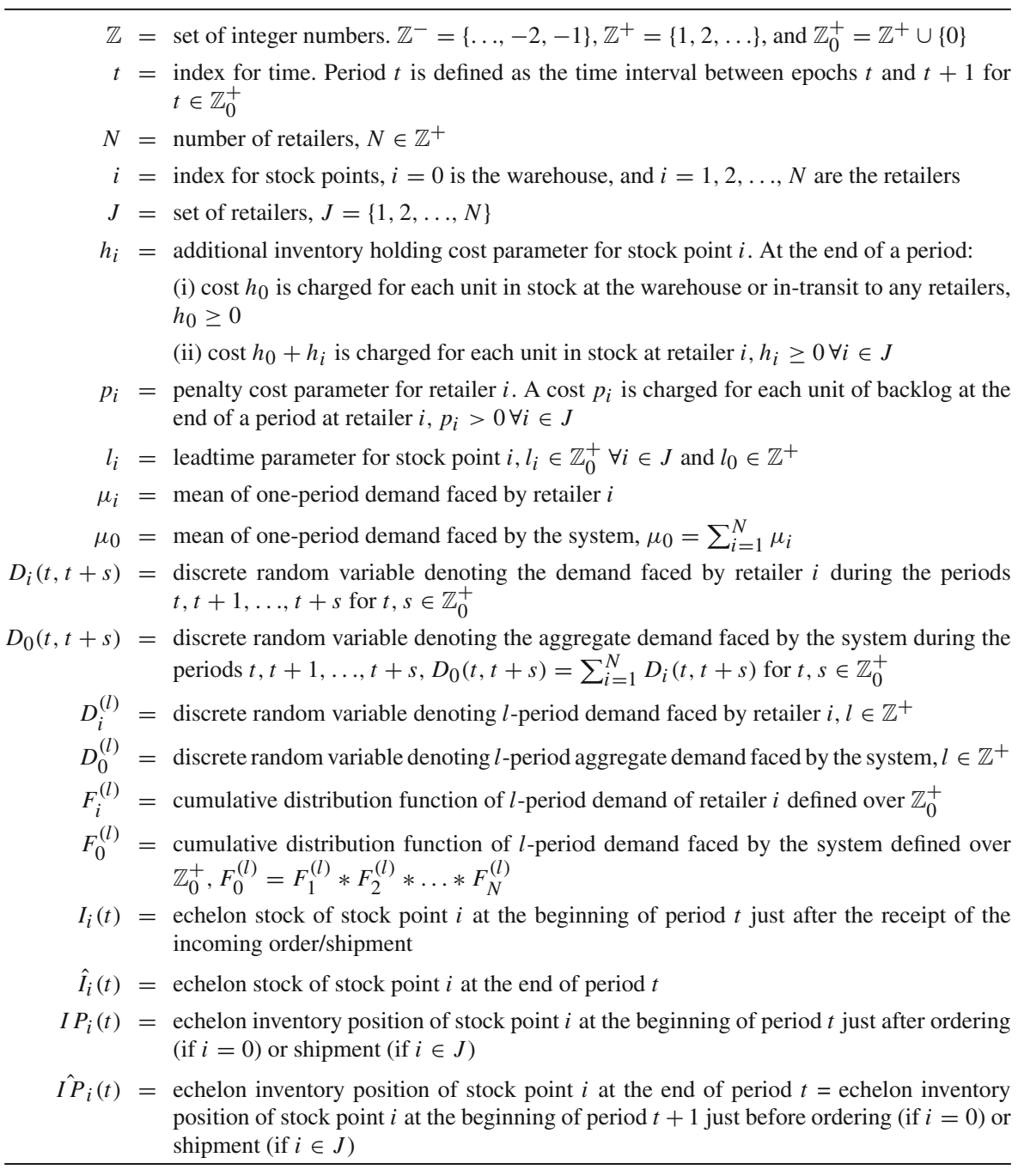

$$
\begin{aligned}
& h_{0}\left(\hat{I}_{0}(t)-\sum_{i \in J} \hat{I}_{i}(t)\right)+\sum_{i \in J}\left(h_{0}+h_{i}\right) \hat{I}_{i}^{+}(t)+\sum_{i \in J} p_{i} \hat{I}_{i}^{-}(t) \\
& =h_{0} \hat{I}_{0}(t)+\sum_{i \in J} h_{i} \hat{I}_{i}^{+}(t)+\sum_{i \in J}\left(h_{0}+p_{i}\right) \hat{I}_{i}^{-}(t) \\
& =h_{0} \hat{I}_{0}(t)+\sum_{i \in J} h_{i} \hat{I}_{i}(t)+\sum_{i \in J}\left(h_{0}+h_{i}+p_{i}\right) \hat{I}_{i}^{-}(t) .
\end{aligned}
$$

We define $h_{0} \hat{I}_{0}(t)$ as the cost attached to the echelon of the warehouse (echelon of stock point 0 ) at the end of period $t$; this cost is denoted by $C_{0}(t)$. We define 


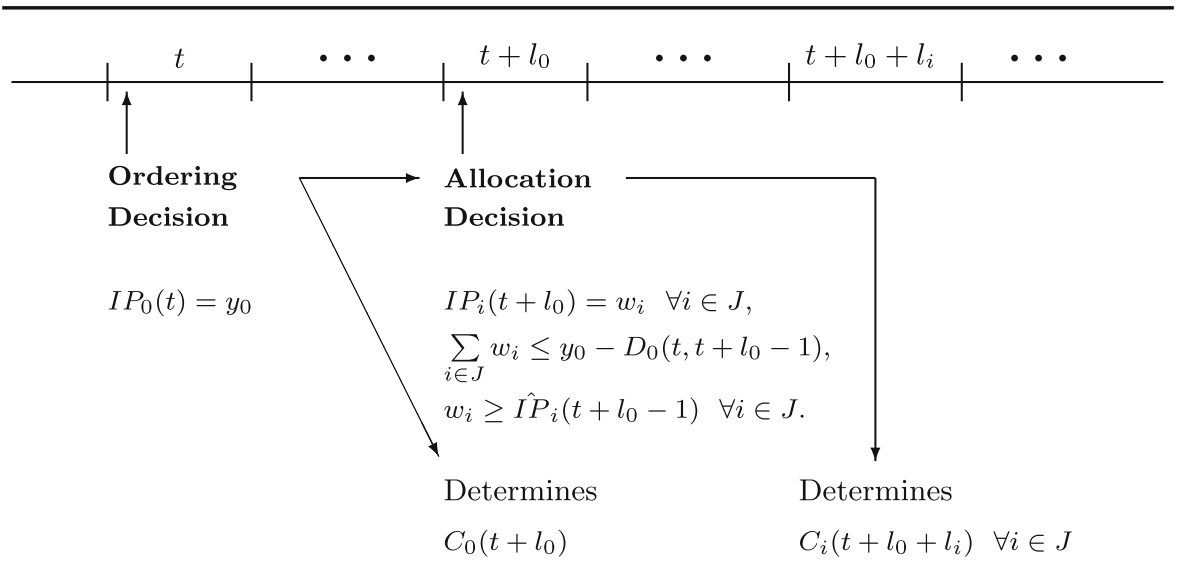

Fig. 1 The consequences of an order placed with the supplier in period $t$

$h_{i} \hat{I}_{i}(t)+\left(h_{0}+h_{i}+p_{i}\right) \hat{I}_{i}^{-}(t)$ as the cost attached to the echelon of retailer $i$ at the end of period $t$, and denote it by $C_{i}(t)$.

Consider the following two decisions, which start with an order given to the supplier in period $t, t \in \mathbb{Z}_{0}^{+}$: (Fig. 1 illustrates the dependence among these decisions and the resulting cost-wise consequences.)

- Ordering decision: Assume that at the beginning of period $t$ the warehouse gives an order that raises the inventory position of the system to some level $y_{0}$, i.e., $I P_{0}(t)=y_{0}$. The order materializes at the beginning of period $t+l_{0}$ and the echelon stock of the warehouse at that epoch is $y_{0}-D_{0}\left(t, t+l_{0}-1\right)$. There are two consequences of the ordering decision:

- It directly determines the expected value of the cost attached to the echelon of the warehouse at the end of period $t+l_{0}$,

$$
\begin{aligned}
\mathbf{E}\left[C_{0}\left(t+l_{0}\right) \mid I P_{0}(t)=y_{0}\right] & =\mathbf{E}\left[h_{0}\left(y_{0}-D_{0}\left(t, t+l_{0}\right)\right)\right] \\
& =h_{0}\left(y_{0}-\left(l_{0}+1\right) \mu_{0}\right) .
\end{aligned}
$$

- It limits the shipment quantities to the retailers. In other words, it puts an upper bound on the level to which one can increase the aggregate echelon inventory positions of the retailers in period $t+l_{0}$,

$$
\sum_{i=1}^{N} I P_{i}\left(t+l_{0}\right) \leq y_{0}-D_{0}\left(t, t+l_{0}-1\right) .
$$

- Allocation decision: At the beginning of period $t+l_{0}$, the system-wide stock is allocated among all stock points. In other words, the shipment quantities to the retailers are determined; as a result, the decision of how much stock to retain at the warehouse is made. At epoch $t+l_{0}$, the inventory position of retailer $i$ is increased to some level $w_{i}$ such that $\sum_{i \in J} w_{i} \leq y_{0}-D_{0}\left(t, t+l_{0}-1\right)$ and $w_{i} \geq \hat{I P} \hat{i}_{i}\left(t+l_{0}\right)$ for all $i \in J$. These decisions directly affect the cost of echelon $i$ at the end of 
period $t+l_{0}+l_{i}$, for all $i \in J$. The expected value of the cost attached to echelon $i$ is

$$
\begin{aligned}
\mathbf{E} & {\left[C_{i}\left(t+l_{0}+l_{i}\right) \mid I P_{i}\left(t+l_{0}\right)=w_{i}\right] } \\
= & \mathbf{E}\left[h_{i}\left(w_{i}-D_{i}\left(t+l_{0}, t+l_{0}+l_{i}\right)\right)\right. \\
& \left.+\left(h_{0}+h_{i}+p_{i}\right)\left(w_{i}-D_{i}\left(t+l_{0}, t+l_{0}+l_{i}\right)\right)^{-}\right] \\
= & h_{i}\left(w_{i}-\left(l_{i}+1\right) \mu_{i}\right)+\left(h_{0}+h_{i}+p_{i}\right) \mathbf{E}\left[\left(w_{i}-D_{i}\left(t+l_{0}, t+l_{0}+l_{i}\right)\right)^{-}\right] .
\end{aligned}
$$

We define the expected costs as a consequence of the ordering and allocation decisions that begin with the warehouse's order given at epoch $t$ as the cycle cost of period $t$ and denote it by $C_{c y c}(t)$ :

$$
C_{c y c}(t)=C_{0}\left(t+l_{0}\right)+\sum_{i \in J} C_{i}\left(t+l_{0}+l_{i}\right)
$$

Let $\Pi$ and $g(\pi)$ denote the set of all ordering policies and the average expected cost of ordering policy $\pi$, respectively. The expected long-run average cost of any policy $\pi \in \Pi$ is simply the average of the expected value of the sum of costs over all cycles:

$$
\begin{aligned}
g(\pi)= & \lim _{T \rightarrow \infty} \frac{1}{T} \mathbf{E}\left[\sum_{t=0}^{T-1} \sum_{i=0}^{N} C_{i}(t)\right] \\
= & \lim _{T \rightarrow \infty} \frac{1}{T} \mathbf{E}\left[\sum_{t=0}^{l_{0}-1} C_{0}(t)+\sum_{t=0}^{l_{0}+l_{i}-1} \sum_{i \in J} C_{i}(t)+\sum_{t=0}^{T-1} C_{c y c}(t)-\sum_{t=T}^{T+l_{0}-1} C_{0}(t)\right. \\
& \left.-\sum_{t=T}^{T+l_{0}+l_{i}-1} \sum_{i \in J} C_{i}(t)\right] \\
= & \lim _{T \rightarrow \infty} \frac{1}{T} \sum_{t=0}^{T-1} \mathbf{E}\left[C_{c y c}(t)\right] .
\end{aligned}
$$

The last equality requires the existence and the finiteness of the limit. Although this may not be the case for any given policy, especially for the ones that do not order enough to satisfy demand, any policy with an underlying Markov process that is unichain meets this requirement. We are interested in such policies. (In the subsequent sections, we show the optimality of base stock policies under the balance assumption. The class of base stock policies is well known to satisfy these necessities.) Thus, the optimization problem that we consider is

$$
\min _{\pi \in \Pi} g(\pi)
$$

The minimization problem given above is intricate since the decisions are highly interdependent. In the next subsection, we discuss the myopic allocation problem and the balance assumption, which yields a relaxation for the optimization problem above. 


\subsection{The allocation decision}

Consider the sequence of decisions and the resulting costs as a result of increasing the inventory position of the system to $y_{0}$ at the beginning of period $t, t \in \mathbb{Z}_{0}^{+}$. Suppose the echelon stock of the warehouse at the beginning of period $t+l_{0}, y_{0}-D_{0}\left(t, t+l_{0}-1\right)$, is distributed among all stock points such that the sum of the expected holding and penalty costs of the retailers in the periods the allocated quantities reach their destinations (i.e., period $t+l_{0}+l_{i}$ for retailer $i$ ) is minimized. This is called myopic allocation because the effect of the allocation decisions on the subsequent periods is not considered. The mathematical formulation of the problem is as follows:

$$
\begin{aligned}
\min _{w_{i}, \forall i \in J} & \sum_{i \in J} \mathbf{E}\left[C_{i}\left(t+l_{0}+l_{i}\right) \mid I P_{i}\left(t+l_{0}\right)=w_{i}\right] \\
\text { s.t. } & \sum_{i \in J} w_{i} \leq y_{0}-D_{0}\left(t, t+l_{0}-1\right) \\
& \hat{I P}_{i}\left(t+l_{0}\right) \leq w_{i} \quad \forall i \in J
\end{aligned}
$$

Both constraints serve for the physical balance of the inventories. While (4) assures that no negative quantity is allocated to the retailers, (3) puts an upper bound on the sum of the allocated quantities. Although myopic allocation allows the allocation decisions to be made independent of the future allocation and ordering decisions, it still depends on previous periods' decisions due to (4). Consider a relaxed version of the myopic allocation problem where (4) is omitted; we refer to this assumption as the balance assumption. In the absence of (4), $C_{c y c}(t)$ depends only on the ordering and allocation decisions that start with an order given by the warehouse in period $t$, not on decisions of other periods. Next, we focus on how to minimize the cycle cost of period $t$.

\subsection{Single cycle analysis}

Consider retailer $i \in J$. Define $G_{i}\left(y_{i}\right)$ as the expected cost attached to echelon $i$ at the end of period $t+l_{i}$ if the inventory position at the beginning of period $t$ is $y_{i}$ :

$$
\begin{aligned}
G_{i}\left(y_{i}\right) & =\mathbf{E}\left[C_{i}\left(t+l_{i}\right) \mid I P_{i}(t)=y_{i}\right] \\
& =h_{i}\left(y_{i}-\left(l_{i}+1\right) \mu_{i}\right)+\left(h_{0}+h_{i}+p_{i}\right) \mathbf{E}\left[\left(D_{i}\left(t, t+l_{i}\right)-y_{i}\right)^{+}\right] .
\end{aligned}
$$

It is straightforward to verify that $G_{i}(\cdot)$ is convex over $\mathbb{Z}$ and $G_{i}\left(y_{i}\right)$ is minimized at all $y_{i} \in Y_{i}^{*}=\left\{\underline{y}_{i}^{*}, \underline{y}_{i}^{*}+1, \ldots, \bar{y}_{i}^{*}\right\}$ where

$$
\begin{aligned}
& \underline{y}_{i}^{*}=\min \left\{y_{i} \mid F_{i}^{\left(l_{i}+1\right)}\left(y_{i}\right) \geq \frac{h_{0}+p_{i}}{h_{0}+h_{i}+p_{i}}\right\}, \quad \text { and } \\
& \bar{y}_{i}^{*}=\min \left\{y_{i} \mid F_{i}^{\left(l_{i}+1\right)}\left(y_{i}\right)>\frac{h_{0}+p_{i}}{h_{0}+h_{i}+p_{i}}\right\} .
\end{aligned}
$$


Notice that if $\underline{y}_{i}^{*}$ satisfies $F_{i}^{\left(l_{i}+1\right)}\left(\underline{y}_{i}^{*}\right)=\frac{h_{0}+p_{i}}{h_{0}+h_{i}+p_{i}}$, then there are multiple optimal values. If $\left\{y_{i} \mid F_{i}^{\left(l_{i}+1\right)}\left(y_{i}\right)>\frac{h_{0}+p_{i}}{h_{0}+h_{i}+p_{i}}\right\}=\emptyset$ then $\bar{y}_{i}^{*}=\infty$. Moreover, (i) $\bar{y}_{i}^{*}=\infty$ if $h_{i}=0$, (ii) $y_{i}^{*}=\infty$ if $h_{i}=0$ and $F_{i}^{(1)}$ has an infinite support.

Let $z_{i}: \overline{\mathbb{Z}}^{i} \rightarrow \mathbb{Z}, i \in J$ be an allocation function such that $z_{i}(a)$ is the portion of $a$ allocated to retailer $i$ for $a \in \mathbb{Z}$. Assume that the system-wide stock at time $t$ is $x \in$ $\mathbb{Z}, I_{0}(t)=x$. The relaxed myopic allocation problem of period $t$ may be rewritten as:

$$
\min _{z_{i}(x) \forall i \in J}\left\{\sum_{i \in J} G_{i}\left(z_{i}(x)\right) \mid \sum_{i \in J} z_{i}(x) \leq x\right\},
$$

where a solution is denoted by $\left\{z_{i}(x)\right\}_{i \in J}$. Next, we discuss how to characterize an optimal solution for the relaxed myopic allocation problem in (6).

Define

$$
\begin{aligned}
\left\{z_{i}^{*}(x)\right\}_{i \in J}= & \text { an optimal solution for }(6) \text { for } x \in \mathbb{Z}, \\
H^{*}(x)= & \text { the optimal objective function value of }(6) \text { for } x \in \mathbb{Z}, \\
& H^{*}(x)=\sum_{i \in J} G_{i}\left(z_{i}^{*}(x)\right) .
\end{aligned}
$$

First, observe that the objective function of (6) consists of $N$ independent components that are convex functions. In the absence of the constraint, the problem is separable and the minimization of each component solves the problem; i.e., $z_{i}^{*}(x) \in Y_{i}^{*}$ for all $i \in J$. In case $x \geq \sum_{i \in J} \underline{y}_{i}^{*}, z_{i}^{*}(x)=\underline{y}_{i}^{*} \forall i \in J$ constitutes an optimal solution, but any other $z_{i}^{*}(x) \in Y_{i}^{*}$ is also possible as long as $\sum_{i \in J} z_{i}^{*}(x) \leq x$.

Second, observe that the constraint should be binding for $x \leq \sum_{i \in J} \underline{y}_{i}^{*}$ :

$$
\text { if } x \leq \sum_{i \in J} \underline{y}_{i}^{*} \text {, then } \sum_{i \in J} z_{i}^{*}(x)=x \text {. }
$$

If $\sum_{i \in J} z_{i}(x)<x$, then it is possible to improve the objective function by allocating one more unit to some retailer $j$ with $z_{j}(x)<\underline{y}_{j}^{*}$ since $\Delta G_{j}\left(y_{j}\right)=G_{j}\left(y_{j}+1\right)-$ $G_{j}\left(y_{j}\right)<0$ for $y_{j}<\underline{y}_{j}^{*}$. This observation can be used to prove the following properties (see Gross (1956) or Saaty (1970, pp. 184-186) for details). For $x<\sum_{i \in J} \underline{y}_{i}^{*}$ and $x \in \mathbb{Z}$ :

P1. A given solution $\left\{z_{i}^{*}(x)\right\}_{i \in J}$ is optimal if and only if:

$$
\Delta G_{i}\left(z_{i}^{*}(x)\right) \geq \Delta G_{j}\left(z_{j}^{*}(x)-1\right) \quad \forall i, j \in J, i \neq j .
$$

P2. Given an optimal solution $\left\{z_{i}^{*}(x)\right\}_{i \in J}$ for $x$, an optimal solution $\left\{z_{i}^{*}(x+1)\right\}_{i \in J}$ for $x+1$ is given by

$$
\begin{aligned}
& z_{k}^{*}(x+1)=z_{k}^{*}(x)+1 \text {, where } k \in\left\{i \in J \mid \Delta G_{i}\left(z_{i}^{*}(x)\right)=\min _{j \in J} \Delta G_{j}\left(z_{j}^{*}(x)\right)\right\} \text {, and } \\
& z_{j}^{*}(x+1)=z_{j}^{*}(x) \quad \forall j \in J \backslash\{k\} .
\end{aligned}
$$


P3. Given an optimal solution $\left\{z_{i}^{*}(x)\right\}_{i \in J}$ for $x$, an optimal solution $\left\{z_{i}^{*}(x-1)\right\}_{i \in J}$ for $x-1$ is given by

$$
\begin{aligned}
& z_{k}^{*}(x-1)=z_{k}^{*}(x)-1, \text { where } k \in\left\{i \in J \mid \Delta G_{i}\left(z_{i}^{*}(x)-1\right)=\max _{j \in J} \Delta G_{j}\left(z_{j}^{*}(x)-1\right)\right\}, \text { and } \\
& z_{j}^{*}(x-1)=z_{j}^{*}(x) \quad \forall j \in J \backslash\{k\} .
\end{aligned}
$$

Properties P2-P3 simply imply that given an optimal solution for $x$, an optimal allocation of $x+1(x-1)$ units can be accomplished by taking the optimal solution for $x$ and giving a retailer $k$ with the smallest $\Delta G_{k}\left(z_{k}(x)\right)$ (largest $\Delta G_{k}\left(z_{k}(x)-1\right)$ ) value one unit more (less). This greedy procedure is also known as the marginal (incremental) allocation, see Fox (1966). An important implication is that if an optimal solution of the relaxed myopic allocation problem is known for some $x \in \mathbb{Z}$, starting from this optimal solution, one can find optimal allocation functions by following the aforementioned greedy procedure.

Property P2 and the fact that the constraint in (6) is binding for $x \leq \sum_{i \in J} y_{i}^{*}$ can be used to show the following properties of the optimal objective function of $(\overline{6})$ :

(i) $\Delta H^{*}(x)<0$ for $x<\sum_{i \in J} \underline{y}_{i}^{*}$,

(ii) $\Delta H^{*}(x)=0$ for $x \geq \sum_{i \in J} \underline{y}_{i}^{*}$,

(iii) $H^{*}(x)$ is convex in $x$.

(See Doğru 2006 for the details.) These properties give the shape of the optimal objective function of the relaxed myopic allocation problem (6) as a function of the amount to allocate. $H^{*}(x)$ is convex, strictly decreasing in the region $\left(-\infty, \sum_{i=1}^{N} \underline{y}_{i}^{*}\right)$, and constant over $\left[\sum_{i=1}^{N} \underline{y}_{i}^{*},+\infty\right)$.

Under the balance assumption, the expected cycle cost of period $t$ is

$$
\begin{aligned}
G_{c y c}\left(y_{0}, \mathbf{z}\right): & =\mathbf{E}\left[C_{0}\left(t+l_{0}\right)+\sum_{i \in J} C_{i}\left(t+l_{0}+l_{i}\right) \mid I P_{0}(t)=y_{0}, \mathbf{z}\right] \\
& =G_{0}\left(y_{0}\right)+\sum_{x=0}^{\infty} \sum_{i \in J} G_{i}\left(z_{i}\left(y_{0}-x\right)\right) \operatorname{Pr}\left\{D_{0}^{\left(l_{0}\right)}=x\right\}
\end{aligned}
$$

for a given $I P_{0}(t)=y_{0}$ and $\mathbf{z}, y_{0} \in \mathbb{Z}$ and $t \in \mathbb{Z}_{0}^{+}$, where

$$
\begin{aligned}
\mathbf{z}= & \text { set of allocation functions, i.e., }\left\{z_{i}\right\}_{i \in J}, \\
G_{0}\left(y_{0}\right)= & \text { expected value of the cost attached to the echelon of the ware- } \\
& \text { house at the end of period } t+l_{0} \text { given } I P_{0}(t)=y_{0} \text { for } y_{0} \in \mathbb{Z}, \text { and } \\
& t \in \mathbb{Z}_{0}^{+}, \text {i.e., } G_{0}\left(y_{0}\right)=\mathbf{E}\left[C_{0}\left(t+l_{0}\right) \mid I P_{0}(t)=y_{0}\right]=h_{0}\left[y_{0}-\right. \\
& \left.\left(l_{0}+1\right) \mu_{0}\right] .
\end{aligned}
$$

Let $\mathbf{z}^{*}$ be a set of optimal allocation functions, i.e., $\left\{z_{i}^{*}\right\}_{i \in J}$ such that $z_{i}^{*}(x)$ is optimal $\forall x \in \mathbb{Z}$ and $\forall i \in J$. It can be shown that regardless of the ordering decision made at the beginning of the cycle, utilizing $\mathbf{z}^{*}$ for allocation leads to expected cycle costs as good as any other set of allocation functions:

$$
G_{c y c}\left(y_{0}, \mathbf{z}^{*}\right) \leq G_{c y c}\left(y_{0}, \mathbf{z}\right) \quad \forall y_{0} \in \mathbb{Z}
$$


Moreover, $G_{c y c}\left(y_{0}, \mathbf{z}^{*}\right)$ is convex in $y_{0}$, and is minimized at all $y_{0} \in Y_{0}^{*}$ such that

$$
\begin{aligned}
& Y_{0}^{*}=\left\{\underline{y}_{0}^{*}, \underline{y}_{0}^{*}+1, \ldots, \bar{y}_{0}^{*}\right\} \text { where } \underline{y}_{0}^{*}=\min \left\{y_{0} \mid \Delta G_{c y c}\left(y_{0}, \mathbf{z}^{*}\right) \geq 0\right\}, \\
& \bar{y}_{0}^{*}=\min \left\{y_{0} \mid \Delta G_{c y c}\left(y_{0}, \mathbf{z}^{*}\right)>0\right\} .
\end{aligned}
$$

Denote a base stock policy by a tuple $\left(y_{0}, \mathbf{z}\right)$, where $y_{0}$ is the target echelon inventory position of the warehouse, and $\left\{z_{i}(x)\right\}_{i \in J}$ are the (state-dependent) target inventory positions of the retailers if the system-wide on-hand stock (state) is $x$. The decisions are made so that, at the beginning of each period $t$ :

- the echelon inventory position of the warehouse is increased up to $y_{0}$, i.e., $I P_{0}(t)=y_{0}$,

- the inventory position of retailer $i$ is raised to $z_{i}\left(I_{0}(t)\right)$, i.e., $I P_{i}(t)=z_{i}\left(I_{0}(t)\right) \forall i \in$ $J$.

Using the convexity of the expected cycle cost function $G_{c y c}\left(\cdot, \mathbf{z}^{*}\right)$, it can be shown that a base stock policy $\left(y_{0}, \mathbf{z}^{*}\right)$ with $y_{0} \in Y_{0}^{*}$ minimizes the average expected cost of a one-warehouse multi-retailer inventory system in an infinite horizon under the balance assumption (see Federgruen and Zipkin 1984b; Doğru 2006).

Remark 1 The balance assumption leads to a relaxation of the original model; thus, $G_{c y c}\left(y_{0}^{*}, z^{*}\right)$ is a lower bound on the long-run expected average cost of the original model. Hence, $G_{c y c}\left(y_{0}^{*}, z^{*}\right)$ is a proxy for the true optimal cost. Further, the optimal policy for the relaxed model can be used to construct a heuristic, see Sect. 3.2 for details.

\section{Newsvendor characterizations}

This section presents our main results for optimal allocation functions and newsvendor inequalities. The existence of nondecreasing optimal allocation functions is essential for the derivation of the newsvendor inequality in Sect. 3.1; hence we begin with our result on this. First, we define

$$
\begin{aligned}
\hat{\mathbf{z}}^{*}= & \text { set of nondecreasing optimal allocation functions, i.e., }\left\{\hat{z}_{i}^{*}\right\}_{i \in J} \\
& \text { such that } \hat{z}_{i}^{*}(x) \text { is optimal and } \Delta \hat{z}_{i}^{*}(x)=\hat{z}_{i}^{*}(x+1)-\hat{z}_{i}^{*}(x) \geq \\
& 0 \forall x \in \mathbb{Z} \text { and } \forall i \in J, \\
\tilde{\mathbf{z}}^{*}= & \text { set of nondecreasing optimal allocation functions with the addi- } \\
& \text { tional property that for all } i \in J \text { with }\left|Y_{i}^{*}\right|>1, \tilde{z}_{i}^{*}(x) \in Y_{i}^{*} \backslash\left\{\bar{y}_{i}^{*}\right\} \\
& \text { for } x>\sum_{i \in J} \underline{y}_{i}^{*} .
\end{aligned}
$$

\section{Theorem 1 There exist nondecreasing optimal allocation functions $\hat{\mathbf{z}}^{*}$.}

Proof Distinguish two cases: (i) $\sum_{i \in J} \underline{y}_{i}^{*}$ is finite, (ii) $\sum_{i \in J} \underline{y}_{i}^{*}$ is infinite. In case (i), $z_{i}^{*}\left(\sum_{i \in J} \underline{y}_{i}^{*}\right)=\underline{y}_{i}^{*} \forall i \in J$ due to the fact that the constraint is binding if the quantity to allocate is less than or equal to $\sum_{i \in J} \underline{y}_{i}^{*}$, see (7). Starting from this optimal solution, optimal solutions for $x<\sum_{i \in J} \underline{y}_{i}^{*}$ can be obtained using property P3 in (10), which 
leads to $z_{i}^{*}(x+1)-z_{i}^{*}(x)=\Delta z_{i}^{*}(x) \in\{0,1\}$ for all $i \in J$. For $x>\sum_{i \in J} \underline{y}_{i}^{*}$, take $z_{i}^{*}(x)=\underline{y}_{i}^{*} \forall i \in J$; as a result $\Delta z_{i}^{*}(x)=0$ for all $i \in J$. In case (ii), an optimal solution $\left\{z_{i}^{*}(x)\right\}_{i \in J}$ for (6) can be determined for some given $x \in \mathbb{Z}$ by Lagrange relaxation (see Everett 1963). Based on $\left\{z_{i}^{*}(x)\right\}_{i \in J}$, optimal solutions for $x+1$ and $x-1$ can be constructed utilizing properties P2 and P3 in (9) and (10), respectively. Continuing in this manner, $z_{i}^{*}(x)$ is determined for all $x \in \mathbb{Z}$ and $i \in J$ such that $\Delta z_{i}^{*}(x) \in\{0,1\}$.

Theorem 1 shows the existence of nondecreasing optimal allocation functions, but not all optimal allocation functions have to be nondecreasing. Consider the case with three identical retailers, i.e., three retailers with identical leadtimes, cost parameters and demand distributions. Define $\bar{z}^{*}(x):=\left(z_{1}^{*}(x), z_{2}^{*}(x), z_{3}^{*}(x)\right)$. There are three optimal alternatives for allocating 4 units: $\bar{z}^{*}(4) \in\{(2,1,1),(1,2,1),(1,1,2)\}$. For $x=5, \bar{z}^{*}(5) \in\{(2,2,1),(1,2,2),(2,1,2)\}$. Given $z^{*}(4)=(2,1,1)$, if one utilizes property $\mathrm{P} 2$, see $(9)$, then $z^{*}(5)$ is $(2,2,1)$ or $(2,1,2)$, which leads to nondecreasing optimal allocation functions at $x=4$. Consider the following optimal allocations: $z^{*}(4)=(2,1,1)$ and $z^{*}(5)=(1,2,2)$. Observe that $\Delta z_{1}^{*}(4)=-1$.

The following corollary follows directly from the proof of Theorem 1 .

\section{Corollary 1 There exist optimal allocation functions $\tilde{\mathbf{z}}^{*}$.}

Proof The additional property in the definition of $\tilde{\mathbf{z}}^{*}$ simply tells that for all the retailers with multiple optima minimizing $G_{i}(\cdot)$ (i.e., for all $i \in J$ with $\left|Y_{i}^{*}\right|>1$ ), $z_{i}^{*}(x) \neq \bar{y}_{i}^{*}$ for all $x>\sum_{i \in J} \underline{y}_{i}^{*}$. In fact, the optimal allocation functions constructed in the proof of Theorem 1 do have the additional property.

Remark 2 There are two causes for an imbalance situation in a one-warehouse multiretailer inventory system. On one hand, the retailers might face disproportionate demands in the previous period and the amount of stock at the warehouse (at the beginning of the current period) is not enough to preclude the allocation of a negative quantity to at least one retailer. On the other hand, imbalance may emanate from decreasing allocation functions. Recall the example of three identical retailers. For some period $t \in \mathbb{Z}_{0}^{+}$, assume that: (i) at the beginning of period $t$, the amount of stock to allocate is 4 , (ii) the amount of stock the warehouse will receive in period $t+1$ is 1 , (iii) $z^{*}(4)=(2,1,1), \bar{z}^{*}(5)=(1,2,2)$. If no demand occurs at any of the retailers in period $\mathrm{t}$, then an imbalance occurs in period $t+1$ due to decreasing $z_{1}^{*}(x)$ for $x=5$. This kind of imbalance can be prevented by using a base stock policy with nondecreasing allocation functions: $\left(y_{0}, \hat{z}^{*}\right)$ with $y_{0} \in Y_{0}^{*}$.

\subsection{Newsvendor inequalities}

The optimality of base stock policies is discussed in Sect. 2. In this subsection, we identify necessary conditions for an optimal warehouse base stock level, which constitute newsvendor inequalities. Define 


$$
\begin{aligned}
\tau\left(y_{0}\right)= & \left(y_{0}-\sum_{i \in J} \underline{y}_{i}^{*}+1\right)^{+} \text {for } y_{0} \in \mathbb{Z}, \\
P_{i}\left(y_{0}, \mathbf{z}\right)= & \begin{array}{l}
\text { probability of no-stockout at retailer } i \text { in period } t+l_{0}+l_{i} \text { given } \\
\mathbf{z}, \text { and } I P_{0}(t)=y_{0} \text { for } y_{0} \in \mathbb{Z} \text { and } t \in \mathbb{Z}_{0}^{+}, \text {i.e. }
\end{array} \\
& P_{i}\left(y_{0}, \mathbf{z}\right)=\sum_{x=0}^{\infty} F_{i}^{\left(l_{i}+1\right)}\left(z_{i}\left(y_{0}-x\right)\right) \operatorname{Pr}\left\{D_{0}^{\left(l_{0}\right)}=x\right\} .
\end{aligned}
$$

Recall from Corollary 1 that there exist nondecreasing allocation functions with the additional property: $\tilde{\mathbf{z}}^{*}$. Next, we derive upper and lower bounds on $\Delta G_{c y c}\left(y_{0}, \tilde{\mathbf{z}}^{*}\right)$.

Lemma 1 For all $i \in J$, and $y_{0} \in \mathbb{Z}$ :

$$
\begin{aligned}
\Delta G_{c y c}\left(y_{0}, \tilde{\mathbf{z}}^{*}\right) \geq & {\left[\left(h_{0}+p_{i}\right)-\left(h_{0}+h_{i}+p_{i}\right) F_{i}^{\left(l_{i}+1\right)}\left(\underline{y}_{i}^{*}\right)\right] F_{0}^{\left(l_{0}\right)}\left(\tau\left(y_{0}\right)-1\right)-p_{i} } \\
& +\left(h_{0}+h_{i}+p_{i}\right)\left[P_{i}\left(y_{0}, \tilde{\mathbf{z}}^{*}\right)\right. \\
& \left.-\sum_{x=\tau\left(y_{0}\right)}^{\infty} \operatorname{Pr}\left\{D_{i}^{\left(l_{i}+1\right)}=\tilde{z}_{i}^{*}\left(y_{0}-x\right)\right\} \operatorname{Pr}\left\{D_{0}^{\left(l_{0}\right)}=x\right\}\right], \\
\Delta G_{c y c}\left(y_{0}, \tilde{\mathbf{z}}^{*}\right) \leq & {\left[\left(h_{0}+p_{i}\right)-\left(h_{0}+h_{i}+p_{i}\right) F_{i}^{\left(l_{i}+1\right)}\left(\underline{y}_{i}^{*}\right)\right] F_{0}^{\left(l_{0}\right)}\left(\tau\left(y_{0}\right)-1\right)-p_{i} } \\
& +\left(h_{0}+h_{i}+p_{i}\right) P_{i}\left(y_{0}, \tilde{\mathbf{z}}^{*}\right) .
\end{aligned}
$$

Proof See the Appendix.

If material availability is always guaranteed by the warehouse then the optimal base stock levels at the retailers are such that the no-stockout probability at each retailer $i \in J$ is at least $\frac{h_{0}+p_{i}}{h_{0}+h_{i}+p_{i}}$, see (5). This constitute the newsvendor characterizations for the retailers. Utilizing this result, similar newsvendor inequalities can also be derived for an optimal warehouse base stock level.

Theorem 2 For each $y_{0} \in Y_{0}^{*}$ :

$$
\begin{aligned}
P_{i}\left(y_{0}, \tilde{\mathbf{z}}^{*}\right) \geq & \frac{p_{i}}{h_{0}+h_{i}+p_{i}} \\
& +\left[F_{i}^{\left(l_{i}+1\right)}\left(\underline{y}_{i}^{*}\right)-\frac{h_{0}+p_{i}}{h_{0}+h_{i}+p_{i}}\right] F_{0}^{\left(l_{0}\right)}\left(\tau\left(y_{0}\right)-1\right) \quad \forall i \in J, \\
P_{i}\left(\underline{y}_{0}^{*}-1, \tilde{\mathbf{z}}^{*}\right)< & \frac{p_{i}}{h_{0}+h_{i}+p_{i}}+\left[F_{i}^{\left(l_{i}+1\right)}\left(\underline{y}_{i}^{*}\right)-\frac{h_{0}+p_{i}}{h_{0}+h_{i}+p_{i}}\right] F_{0}^{\left(l_{0}\right)}\left(\tau\left(\underline{y}_{0}^{*}\right)-2\right) \\
& +\sum_{x=\tau\left(\underline{y}_{0}^{*}\right)-1}^{\infty} \operatorname{Pr}\left\{D_{i}^{\left(l_{i}+1\right)}=\tilde{z}_{i}^{*}\left(\underline{y}_{0}^{*}-1-x\right)\right\} \operatorname{Pr}\left\{D_{0}^{\left(l_{0}\right)}=x\right\} \quad \forall i \in J .
\end{aligned}
$$


Proof These inequalities follow directly from the properties that $\Delta G_{c y c}\left(\underline{y}_{0}^{*}, \tilde{\mathbf{z}}^{*}\right) \geq 0$ and $\Delta G_{c y c}\left(\underline{y}_{0}^{*}-1, \tilde{\mathbf{z}}^{*}\right)<0$, and the result of Lemma 1 .

Theorem 2 implies that an optimal base stock level of the warehouse leads to a no-stockout probability at each retailer $i \in J$, which is at least $\frac{p_{i}}{h_{0}+h_{i}+p_{i}}$. Note that $F_{0}^{\left(l_{0}\right)}\left(\tau\left(y_{0}\right)-1\right)$ in $(14)$ corresponds to the probability that retailers can reach inventory positions $\underline{y}_{i}^{*}$ via shipments from the warehouse (i.e., there is no shortage at the warehouse). $F_{i}^{\left(l_{i}+1\right)}\left(\underline{y}_{i}^{*}\right)-\frac{h_{0}+p_{i}}{h_{0}+h_{i}+p_{i}}$ is the overshoot from the target newsvendor level for retailer $i$ due to discreteness. In case of continuous demand, there is no overshoot; moreover, the newsvendor inequalities for the retailers and (14) can be satisfied with equality. Thus, (14) can be streamlined as $P_{i}\left(y_{0}^{*}, \tilde{\mathbf{z}}^{*}\right)=\frac{p_{i}}{h_{0}+h_{i}+p_{i}}$ for all $i \in J$ (cf. Diks and de Kok 1998).

Corollary $2 P_{i}\left(y_{0}, \tilde{\mathbf{z}}^{*}\right) \geq \frac{p_{i}}{h_{0}+h_{i}+p_{i}} \forall i \in J, \forall y_{0} \in Y_{0}^{*}$.

Proof Result follows directly from (14) and the definition of $\underline{y}_{i}^{*}$.

The newsvendor inequalities derived in Theorem 2 allow us to see the following direct relations between the holding cost parameters and the base stock levels under an optimal policy.

Corollary 3 If there exists a retailer $i \in J$ with $h_{i}=0$ and an infinite support for its demand distribution $F_{i}^{(1)}$, then the warehouse becomes a cross-docking point under an optimal policy.

Proof Note that $\underline{y}_{i}^{*}=\infty$, see (5). Thus, in each period, all available stock at the warehouse is allocated to the retailers under an optimal policy.

Lemma 2 (i) If $h_{0}=0$ then the inventory position of retailer $i$ can always be increased to at least $\underline{y}_{i}^{*}$ for all $i \in J$ under an optimal policy $\left(y_{0}, \tilde{\mathbf{z}}^{*}\right)$ with $y_{0} \in Y_{0}^{*}$.

(ii) If $h_{0}=0$ and there is at least one retailer $i \in J$ with an infinite support for its demand distribution $F_{i}^{(1)}$, then $\underline{y}_{0}^{*}=\infty$ under an optimal policy $\left(y_{0}, \tilde{\mathbf{z}}^{*}\right), y_{0} \in$ $Y_{0}^{*}=\{\infty\}$. Thus, infinite stock is kept at the warehouse.

Proof See the Appendix.

For $N=1$, the model reduces to a two-echelon serial system facing discrete demand. The newsvendor inequalities discussed in this subsection coincide with the results of Doğru (2006) if the fixed order quantities in their model are one, i.e., $Q_{1}=$ $Q_{2}=1$.

\subsection{Computational issues}

The results so far can be used to develop an efficient optimization scheme. The general line is reminiscent of the technique developed for serial systems by 
Clark and Scarf (1960). First, $y_{i}^{*} \forall i \in J$ are calculated utilizing (5). Second, following the arguments in the proof of Theorem 1 and using properties P2 and P3, see (9) and (10), $\tilde{\mathbf{z}}^{*}$ is determined. Finally, a simple search procedure is used to find $\underline{y}_{0}^{*}$; the details are as follows. Consider a retailer $i \in J$, preferably one with $\left|Y_{i}^{*}\right|>1$. Start the search at $y_{0}$ for which $P_{i}\left(y_{0}, \tilde{\mathbf{z}}^{*}\right) \geq \frac{p_{i}}{h_{0}+h_{i}+p_{i}}$ for the first time. Unless $\Delta G_{c y c}\left(y_{0}, \tilde{\mathbf{z}}^{*}\right) \geq 0$, increase $y_{0}$ by a suitable step size (depending on the distribution of demand at retailer $i)$ until $\Delta G_{c y c}\left(y_{0}, \tilde{\mathbf{z}}^{*}\right) \geq 0$. Initiate a bisection procedure and terminate it when $\underline{y}_{0}^{*}$ is determined. Once $\underline{y}_{0}^{*}$, and $\tilde{\mathbf{z}}^{*}$ are obtained the values are substituted into (11), which gives the optimal long-run average cost of the one-warehouse multi-retailer inventory system under the balance assumption.

The optimal solution for the relaxed model may be infeasible due to negative shipment quantities. If the optimal base stock levels of the lower bound model are coupled with myopic allocation without the balance assumption, given in (2)-(4), it leads to a feasible heuristic policy. It is not easy to determine the average expected cost of this policy analytically, but simulation can be used to estimate it.

The balance assumption is the relaxation of a set of constraints in the original model, hence, it yields a lower bound model, which can be solved exactly, see Sect. 2.3. This gives us a lower bound for the optimal cost of the original model, which is $G_{c y c}\left(y_{0}, \tilde{\mathbf{z}}^{*}\right)$. An upper bound for the true optimal cost is obtained by simulating the optimal policy for the relaxed problem with a slightly modified allocation rule, which is discussed above. Doğru et al. (2010) use the relative gap between the upper and lower bound as a measure to assess the impact of the balance assumption. Their extensive numerical experiments identify which parameter settings yield to small gaps; a small gap implies that both the lower bound is tight and the performance of the heuristic policy is close to the optimal. They also identify many practically relevant settings under which the balance assumption yields large gaps. For these instances, either the lower bound is poor or the LB heuristic policy is far from optimal, or both.

\section{Conclusion}

This paper considers a one-warehouse multi-retailer inventory system facing discrete demands. Under the balance assumption, we show that the optimal base stock levels satisfy newsvendor characterizations. These characterizations are appealing because they are easy to explain to nonmathematical oriented people like managers and MBA students, and help intuition development by providing direct relation between cost and optimal policy parameters. Finally, the results are used to develop an efficient computational scheme for the determination of optimal base stock levels. We believe that newsvendor characterizations can be derived for more multi-echelon structures, and this will be a direction for further research.

Acknowledgments This research had been conducted while the first author was a PhD student at The Department of Industrial Engineering and Innovation Sciences, Technische Universiteit Eindhoven. He was supported by NWO (National Science Foundation of the Netherlands) grant 425-10-004. 


\section{Appendix}

Proof of Lemma 1 For all $y_{0} \in \mathbb{Z}$ :

$$
\begin{aligned}
\Delta G_{c y c}\left(y_{0}, \mathbf{z}^{*}\right)= & \Delta G_{0}\left(y_{0}\right)+\sum_{x=0}^{\infty} \Delta H^{*}\left(y_{0}-x\right) \operatorname{Pr}\left\{D_{0}^{\left(l_{0}\right)}=x\right\} \\
= & h_{0}+\sum_{x=0}^{\infty} \Delta H^{*}\left(y_{0}-x\right) \operatorname{Pr}\left\{D_{0}^{\left(l_{0}\right)}=x\right\} . \\
= & h_{0}+\sum_{x=\tau\left(y_{0}\right)}^{\infty} \Delta H^{*}\left(y_{0}-x\right) \operatorname{Pr}\left\{D_{0}^{\left(l_{0}\right)}=x\right\} \\
= & h_{0}+\sum_{x=\tau\left(y_{0}\right)}^{\infty}\left[\sum_{j \in J} G_{j}\left(\tilde{z}_{j}^{*}\left(y_{0}+1-x\right)\right)\right. \\
& \left.-\sum_{j \in J} G_{j}\left(\tilde{z}_{j}^{*}\left(y_{0}-x\right)\right)\right] \operatorname{Pr}\left\{D_{0}^{\left(l_{0}\right)}=x\right\} \\
= & h_{0}+\sum_{x=\tau\left(y_{0}\right)}^{\infty} \min _{j \in J}\left\{\Delta G_{j}\left(\tilde{z}_{j}^{*}\left(y_{0}-x\right)\right)\right\} \operatorname{Pr}\left\{D_{0}^{\left(l_{0}\right)}=x\right\} .
\end{aligned}
$$

Note that for $x \geq \tau\left(y_{0}\right), x \in \mathbb{Z}$ :

$$
\Delta G_{i}\left(\tilde{z}_{i}^{*}\left(y_{0}-x\right)-1\right) \leq \min _{j \in J}\left\{\Delta G_{j}\left(\tilde{z}_{j}^{*}\left(y_{0}-x\right)\right)\right\} \leq \Delta G_{i}\left(\tilde{z}_{i}^{*}\left(y_{0}-x\right)\right) \quad \forall i \in J
$$

While the upper bound in (16) is obvious, lower bound follows from property P1 given in (8). Substituting (16) into (15) yields

$$
\begin{aligned}
& h_{0}+\sum_{x=\tau\left(y_{0}\right)}^{\infty} \Delta G_{i}\left(\tilde{z}_{i}^{*}\left(y_{0}-x\right)-1\right) \operatorname{Pr}\left\{D_{0}^{\left(l_{0}\right)}=x\right\} \leq \Delta G_{c y c}\left(y_{0}, \tilde{\mathbf{z}}^{*}\right) \leq h_{0} \\
& +\sum_{x=\tau\left(y_{0}\right)}^{\infty} \Delta G_{i}\left(\tilde{z}_{i}^{*}\left(y_{0}-x\right)\right) \operatorname{Pr}\left\{D_{0}^{\left(l_{0}\right)}=x\right\},
\end{aligned}
$$

for all $i \in J$. The lower bound may be rewritten in terms of $P_{i}\left(y_{0}, \tilde{\mathbf{z}}^{*}\right)$, which is defined in (13):

$$
\begin{aligned}
& h_{0}+\sum_{x=\tau\left(y_{0}\right)}^{\infty} \Delta G_{i}\left(\tilde{z}_{i}^{*}\left(y_{0}-x\right)-1\right) \operatorname{Pr}\left\{D_{0}^{\left(l_{0}\right)}=x\right\} \\
& =h_{0}+\sum_{x=\tau\left(y_{0}\right)}^{\infty}\left[\left(h_{0}+h_{i}+p_{i}\right) F_{i}^{\left(l_{i}+1\right)}\left(\tilde{z}_{i}^{*}\left(y_{0}-x\right)-1\right)-\left(h_{0}+p_{i}\right)\right] \operatorname{Pr}\left\{D_{0}^{\left(l_{0}\right)}=x\right\}
\end{aligned}
$$




$$
\begin{aligned}
= & \left(h_{0}+p_{i}\right) F_{0}^{\left(l_{0}\right)}\left(\tau\left(y_{0}\right)-1\right)-p_{i} \\
& +\sum_{x=\tau\left(y_{0}\right)}^{\infty}\left[\left(h_{0}+h_{i}+p_{i}\right) F_{i}^{\left(l_{i}+1\right)}\left(\tilde{z}_{i}^{*}\left(y_{0}-x\right)-1\right)\right] \operatorname{Pr}\left\{D_{0}^{\left(l_{0}\right)}=x\right\} \\
= & \left(h_{0}+p_{i}\right) F_{0}^{\left(l_{0}\right)}\left(\tau\left(y_{0}\right)-1\right)-p_{i} \\
& +\left(h_{0}+h_{i}+p_{i}\right) \sum_{x=\tau\left(y_{0}\right)}^{\infty}\left(F_{i}^{\left(l_{i}+1\right)}\left(\tilde{z}_{i}^{*}\left(y_{0}-x\right)\right)\right. \\
& \left.-\operatorname{Pr}\left\{D_{i}^{\left(l_{i}+1\right)}=\tilde{z}_{i}^{*}\left(y_{0}-x\right)\right\}\right) \operatorname{Pr}\left\{D_{0}^{\left(l_{0}\right)}=x\right\} \\
= & \left(h_{0}+p_{i}\right) F_{0}^{\left(l_{0}\right)}\left(\tau\left(y_{0}\right)-1\right)-p_{i} \\
& +\left(h_{0}+h_{i}+p_{i}\right)\left[P_{i}\left(y_{0}, \tilde{\mathbf{z}}^{*}\right)-\sum_{x=0}^{\tau\left(y_{0}\right)-1} F_{i}^{\left(l_{i}+1\right)}\left(\tilde{z}_{i}^{*}\left(y_{0}-x\right)\right) \operatorname{Pr}\left\{D_{0}^{\left(l_{0}\right)}=x\right\}\right. \\
& \left.-\sum_{x=\tau\left(y_{0}\right)}^{\infty} \operatorname{Pr}\left\{D_{i}^{\left(l_{i}+1\right)}=\tilde{z}_{i}^{*}\left(y_{0}-x\right)\right\} \operatorname{Pr}\left\{D_{0}^{\left(l_{0}\right)}=x\right\}\right] .
\end{aligned}
$$

Recall from (5) that $F_{i}^{\left(l_{i}+1\right)}\left(y_{i}\right)=F_{i}^{\left(l_{i}+1\right)}\left(\underline{y}_{i}^{*}\right)$ for $y_{i} \in Y_{i}^{*} \backslash\left\{\bar{y}_{i}^{*}\right\}$. Hence, the expres$\operatorname{sion} \sum_{x=0}^{\tau\left(y_{0}\right)-1} F_{i}^{\left(l_{i}+1\right)}\left(\tilde{z}_{i}^{*}\left(y_{0}-x\right)\right) \operatorname{Pr}\left\{D_{0}^{\left(l_{0}\right)}=x\right\}$ reduces to $F_{i}^{\left(l_{i}+1\right)}\left(\underline{y}_{i}^{*}\right) F_{0}^{\left(l_{0}\right)}\left(\tau\left(y_{0}\right)-\right.$ 1) and rearranging the terms yields

$$
\begin{aligned}
& h_{0}+\sum_{x=\tau\left(y_{0}\right)}^{\infty} \Delta G_{i}\left(\tilde{z}_{i}^{*}\left(y_{0}-x\right)-1\right) \operatorname{Pr}\left\{D_{0}^{\left(l_{0}\right)}=x\right\} \\
& =\left[\left(h_{0}+p_{i}\right)-\left(h_{0}+h_{i}+p_{i}\right) F_{i}^{\left(l_{i}+1\right)}\left(\underline{y}_{i}^{*}\right)\right] F_{0}^{\left(l_{0}\right)}\left(\tau\left(y_{0}\right)-1\right)-p_{i} \\
& +\left(h_{0}+h_{i}+p_{i}\right)\left[P_{i}\left(y_{0}, \tilde{\mathbf{z}}^{*}\right)-\sum_{x=\tau\left(y_{0}\right)}^{\infty} \operatorname{Pr}\left\{D_{i}^{\left(l_{i}+1\right)}=\tilde{z}_{i}^{*}\left(y_{0}-x\right)\right\} \operatorname{Pr}\left\{D_{0}^{\left(l_{0}\right)}=x\right\}\right] .
\end{aligned}
$$

Similarly, the upper bound can be expressed in terms of $P_{i}\left(y_{0}, \tilde{\mathbf{z}}^{*}\right)$ :

$$
\begin{aligned}
& h_{0}+\sum_{x=\tau\left(y_{0}\right)}^{\infty} \Delta G_{i}\left(\tilde{z}_{i}^{*}\left(y_{0}-x\right)\right) \operatorname{Pr}\left\{D_{0}^{\left(l_{0}\right)}=x\right\} \\
& =\left[\left(h_{0}+p_{i}\right)-\left(h_{0}+h_{i}+p_{i}\right) F_{i}^{\left(l_{i}+1\right)}\left(\underline{y}_{i}^{*}\right)\right] F_{0}^{\left(l_{0}\right)}\left(\tau\left(y_{0}\right)-1\right)-p_{i} \\
& \quad+\left(h_{0}+h_{i}+p_{i}\right) P_{i}\left(y_{0}, \tilde{\mathbf{z}}^{*}\right) .
\end{aligned}
$$


Proof of Lemma $2 \quad$ (i) Choose retailer $i \in J$ such that $\tilde{z}_{i}^{*}\left(\sum_{i \in J} \underline{y}_{i}^{*}-1\right)=\underline{y}_{i}^{*}-1$. From (13) and (14), for each $y_{0} \in Y_{0}^{*}$ :

$$
\begin{aligned}
P_{i}\left(y_{0}, \tilde{\mathbf{z}}^{*}\right)= & \sum_{x=0}^{\tau\left(y_{0}\right)-1} F_{i}^{\left(l_{i}+1\right)}\left(\tilde{z}_{i}^{*}\left(y_{0}-x\right)\right) \operatorname{Pr}\left\{D_{0}^{\left(l_{0}\right)}=x\right\} \\
& +\sum_{x=\tau\left(y_{0}\right)}^{\infty} F_{i}^{\left(l_{i}+1\right)}\left(\tilde{z}_{i}^{*}\left(y_{0}-x\right)\right) \operatorname{Pr}\left\{D_{0}^{\left(l_{0}\right)}=x\right\} \\
\geq & \frac{p_{i}}{h_{i}+p_{i}}+\left(F_{i}^{\left(l_{i}+1\right)}\left(\underline{y}_{i}^{*}\right)-\frac{p_{i}}{h_{i}+p_{i}}\right) F_{0}^{\left(l_{0}\right)}\left(\tau\left(y_{0}\right)-1\right),
\end{aligned}
$$

which can be rewritten as

$$
\sum_{x=\tau\left(y_{0}\right)}^{\infty} F_{i}^{\left(l_{i}+1\right)}\left(\tilde{z}_{i}^{*}\left(y_{0}-x\right)\right) \operatorname{Pr}\left\{D_{0}^{\left(l_{0}\right)}=x\right\} \geq \frac{p_{i}}{h_{i}+p_{i}}\left(1-F_{0}^{\left(l_{0}\right)}\left(\tau\left(y_{0}\right)-1\right)\right)
$$

using the property that $F_{i}^{\left(l_{i}+1\right)}\left(\tilde{z}_{i}^{*}(x)\right)=F_{i}^{\left(l_{i}+1\right)}\left(\underline{y}_{i}^{*}\right)$ for $x \geq \sum_{i \in J} \underline{y}_{i}^{*}, x \in \mathbb{Z}$. Further, the inequality in (17) may be rewritten as

$$
\sum_{x=\tau\left(y_{0}\right)}^{\infty} F_{i}^{\left(l_{i}+1\right)}\left(\tilde{z}_{i}^{*}\left(y_{0}-x\right)\right) \operatorname{Pr}\left\{D_{0}^{\left(l_{0}\right)}=x\right\} \geq \sum_{x=\tau\left(y_{0}\right)}^{\infty} \frac{p_{i}}{h_{i}+p_{i}} \operatorname{Pr}\left\{D_{0}^{\left(l_{0}\right)}=x\right\} .
$$

From $\tilde{z}_{i}^{*}\left(\sum_{i \in J} \underline{y}_{i}^{*}-1\right)=\underline{y}_{i}^{*}-1$ and (5), $F_{i}^{\left(l_{i}+1\right)}\left(\tilde{z}_{i}^{*}\left(y_{0}-x\right)\right)<\frac{p_{i}}{h_{i}+p_{i}}$ for $x \geq$ $\tau\left(y_{0}\right)$. Thus, the inequality in (18) can only be satisfied if $\operatorname{Pr}\left\{D_{0}^{\left(l_{0}\right)} \geq \tau\left(y_{0}\right)\right\}=$ 0 , i.e., $F_{0}^{\left(l_{0}\right)}\left(\tau\left(y_{0}\right)-1\right)=1$. This implies that $y_{0} \in Y_{0}^{*}$ is greater than or equal to any possible realization of $D_{0}^{\left(l_{0}\right)}$ plus $\sum_{i \in J} \underline{y}_{i}^{*}$.

(ii) An infinite support for $F_{i}^{(1)}, i \in J$, implies that there is also an infinite support for $F_{0}^{(1)}$. From part (i), $F_{0}^{\left(l_{0}\right)}\left(\tau\left(y_{0}\right)-1\right)=1$ for $y_{0} \in Y_{0}^{*}$ can only be attained if $\underline{y}_{0}^{*}=\infty$.

\section{References}

Aviv Y, Federgruen A (2001) Capacitated multi-item inventory systems with random and seasonally fluctuating demands: implications for postponement strategies. Manag Sci 47:512-531

Axsäter S (2003) Supply Chain Operations: Serial and Distribution Inventory Systems. In: de Kok AG, Graves SC (eds) Handbook in operations research and management science, volume 11: design and analysis of supply chains. Elsevier, Amsterdam pp 525-559

Chen F (2000) Optimal policies for multi-echelon inventory problems with batch ordering. Oper Res 48:376-389

Chen F, Zheng YF (1994) Lower bounds for multi-echelon stochastic inventory problems. Manag Sci 40:1426-1443

Clark AJ, Scarf H (1960) Optimal policies for a multi-echelon inventory problem. Manag Sci 6:475-490 de Kok AG (1989) A moment-iteration method for approximating the waiting-time characteristics of the GI/G/1 queue. Prob Eng Inf Sci 3:273-287 
de Kok AG, Fransoo JC (2003) Planning supply chain operations: Definition and comparison of planning concepts. In: de Kok AG, Graves SC (eds) Handbook in operations research and management science, volume 11: design and analysis of supply chains. Elsevier, Amsterdam pp 597-675

Diks EB, de Kok AG (1998) Optimal control of a divergent $N$-echelon inventory system. Eur J Oper Res 111:75-97

Doğru MK (2006) Optimal control of one-warehouse multi-retailer systems: an assessment of the balance assumption. Ph.D. thesis, Beta Research School, D77, Technische Universiteit Eindhoven, Eindhoven

Doğru MK, van Houtum GJ, de Kok AG (2008) Newsboy characterizations for optimal reorder levels of serial inventory systems with fixed batch sizes. Oper Res Lett 36:551-556

Doğru MK, de Kok AG, van Houtum GJ (2010) A numerical study on the effect of the balance assumption in one-warehouse multi-retailer inventory systems. Flex Serv Manuf J 21:114-147

Eppen G, Schrage L (1981) Centralized Ordering Policies in a Multi-Warehouse System with Lead Times and Random Demand. In: Schwarz LB (ed) Multi-level production-inventory control systems: theory and practice. North-Holland, Amsterdam pp 51-67

Everett H (1963) Generalized lagrange multiplier method for solving problems of optimum allocation of resources. Oper Res 11:399-417

Federgruen A, Zipkin P (1984a) Approximations of dynamic, multi-location production and inventory problems. Manag Sci 30:69-84

Federgruen A, Zipkin P (1984b) Computational issues in an infinite-horizon, multi-echelon inventory model. Oper Res 32:818-836

Fox B (1966) Discrete optimization via marginal analysis. Manag Sci 13:210-216

Gross OA (1956) Notes on linear programming: a class of discrete-type minimization problems. Tech. rept. RM-1644. RAND Corporation, Santa Monica

Lee HL, Tang CS (1997) Modelling the costs and benefits of delayed product differentiation. Manag Sci 43:40-53

Rogers DF, Tsubakitani S (1991) Inventory positioning/partitioning for backorders optimization for a class of multi-echelon inventory problems. Decis Sci 22:536-558

Saaty TL (1970) Optimization in integers and related extremal problems. McGraw-Hill, New York

van Houtum GJ (2006) Multi-echelon production/inventory systems: Optimal policies, heuristics, and algorithms. In: Johnson MP, Norman B, Secomandi N (eds) INFORMS tutorials in operations research series. INFORMS, Hanover pp 163-199

van Houtum GJ, Zijm WHM (1991) Computational procedures for stochastic multi-echelon production systems. Int J Prod Econ 23:223-237

van Houtum GJ, Scheller-Wolf A, Yi J (2007) Opimal control of serial inventory systems with fixed replenishment intervals. Oper Res 55:674-687

Wijngaard J (1982) On aggregation in production planning. Eng Costs Prod Plan 6:259-265

Zipkin P (2000) Foundations of inventory management. McGraw-Hill, Boston 